\title{
Implementation of a methodology for evaluation of the reliability of mechatronic systems
}

\author{
N. HAMMOUDA, G. HABCHI, C. BARTHOD, J. LOTTIN \\ Univ. Savoie, SYMME, F-74000 Annecy \\ Naima.chouket@univ-savoie.fr \\ Georges.habchi@univ-savoie.fr \\ Christine.barthod@univ-savoie.fr \\ Jacques.lottin@univ-savoie.fr
}

\author{
O. DUVERGER \\ CETIM, Maison de la Mécatronique \\ 7, chemin de Bellevue - BP 80439 \\ 74944 Annecy-le-Vieux Cedex \\ Olivier.Duverger@cetim.fr
}

\begin{abstract}
Reliability is one of the most important parameters of dependability. It is addressed whenever we want reliable, available and safe systems. Indeed, reliability acts on the availability and security of systems. It must be considered throughout the overall life cycle of the product or system (design, manufacturing, operation).

The objective of this communication is to build an overall approach of reliability for mechatronic systems since they are complex and multi-technology integrating several properties such as: dynamic, hybrid, reconfigurable, and interactive.
\end{abstract}

Keywords - methodology, reliability, mechatronics system, modeling, simulation, qualitative and quantitative analysis, interactions, mission profile

\section{INTRODUCTION}

The development of mechatronic systems over the past twenty years can be considered as a revolution for the industry. The use of these systems is widespread fast and now affects almost all sectors of industry. Before addressing the "reliability" is the central theme of this paper, let's take a ride background to identify what the literature meant by the term "Mechatronics". Indeed, several definitions exist. Let's quote the main non-exhaustive. Mechatronics International Journal published a definition of this word for the first time in 1991: "Mechatronics in its fundamental form can be regarded as the fusion of mechanical and electrical disciplines in modern engineering process. It is a relatively new concept to the design of systems, devices and products aimed at achieving optimal balance between year basic mechanical structures and its overall control" [8]. In 1996, the international journal IEEE Transactions on Mechatronics defined it as followed: "Mechatronics is the synergetic combination of mechanical engineering with electronics and intelligent computer control in the design and manufacturing of industrial products and processes" [9]. The IFAC Technical Committee on Mechatronic Systems chose the following definition in 2000: "Many technical processes and products in the area of mechanical and electrical engineering show an increasing integration of mechanics with electronics and information processing. This integration is between the components (hardware) and the information driven function (software), resulting and in integrated systems called expired mechatronic systems" [5].

According to standard NF E-010, mechatronics is defined as an "approach to integration synergy of mechanics, electronics, automation and information technology in the design and manufacture of a product to increase and / or optimize its functionality "[10].

From these various approaches, we proposed to define mechatronic systems as systems that are characterized by its kind of behaviour: hybrid, dynamic, reconfigurable and interactive i.e. by the existence of interactions between the various components that may be due in part to the integration of several technologies. Hybrid systems are systems characterized by the presence of continuous phenomena and discrete event [6]. Dynamical systems are characterized by functional relationships between components that constitute them. If these relations remain frozen throughout the mission system, the system is said to be static. If, however, these relationships change during the mission, the system is said to be dynamic [5]. The interactive nature of a system is defined by the existence of physical and/or functional interactions between the components of the system. Finally, reconfigurable systems are systems that can change their internal structures to ensure the realization of the function [5]. All these characteristics must be taken into account when developing the methodology to estimate the reliability of these systems.

One of the major problems encountered during the design phase of mechatronic systems is the evaluation of the reliability using an overall approach that takes into account all system technologies and cultures of the organization. Indeed, although the existing methods related to the field of reliability are numerous, they are mainly dedicated to electronic and mechanical technologies, but rarely for the software. But more importantly, they are applied independently to these technologies and related components without considering the integration synergy for product as a whole. In recent years, few studies have addressed this scientific theme; we cite two 
main theses treated assessing the reliability of mechatronic systems by A.Demri [11] and A.Mihalache [1]. A. Mihalache has developed a methodology to evaluate the reliability prediction, experimental and operational while A. Demri focused only on reliability prediction taking into account the dynamic of these systems. Although this work is important for the scientific community, they still ignored the other characters such as reconfigurable, hybrid or interactive who are, our sense, essential characteristics and necessary to evaluate the reliability of mechatronic systems. At present, despite the work made over the last decade, there is no knowledge for an overall approach studying the reliability of a mechatronic system and taking into account all the characters mentioned before and the interactions generated between the different technological areas. In addition, due to the complexity of the study area, the gaps in this field are numerous and affect many aspects: many scattering methods, several different technologies, different phases in the life of the system, regardless of the qualitative and quantitative aspects, diversity of available scientific tools, study objectives, etc.

It is on this issue that we propose an overall approach studying the reliability of mechatronic systems during the design phase while taking into account the different characters, the interactions between the different technologies, and the needs of the industrial sector.

The paper is organized as follows. First, we present in Section II the proposed methodology for evaluation of the predictive reliability. Paragraphs A to $G$ detail the different steps of this methodology. Finally, in Section III we propose a conclusion for this work.

\section{METHODOLOGY FOR EVALUATION THE PREDICTIVE RELIABILITY OF MECHATRONIC SYSTEMS}

The proposed methodology of the predictive reliability of mechatronic systems must take into account the following points:

- The intrinsic characteristics of mechatronic systems (dynamic, hybrid, reconfigurable, multi-technology, interactive)

- The mission profile

- Expert opinion

- Qualitative analysis (functional, organic and dysfunctional analysis, functional and dysfunctional modelling)

- Quantitative analysis (modelling and simulation)

- The study of the system reliability, sub-systems and components

- The lack of reliability in the industrial sector (especially technological interactions)

Figure 1 present the proposed methodology with steps to evaluate the predictive reliability of mechatronic systems.

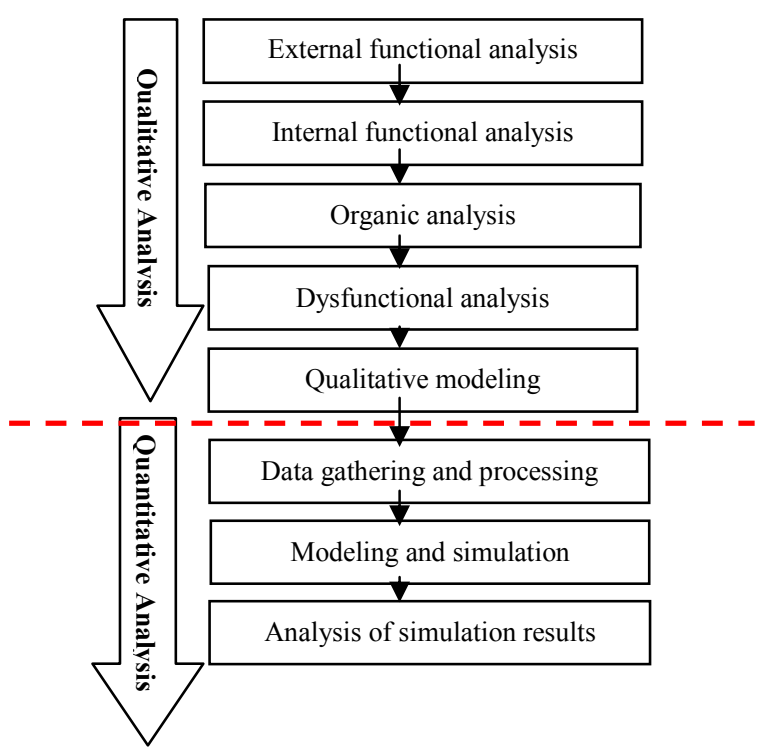

Fig. 1: Overall Methodology evaluating the predictive reliability of mechatronic systems.

We describe in the following paragraphs the main steps of this methodology.

\section{A. External functional analysis}

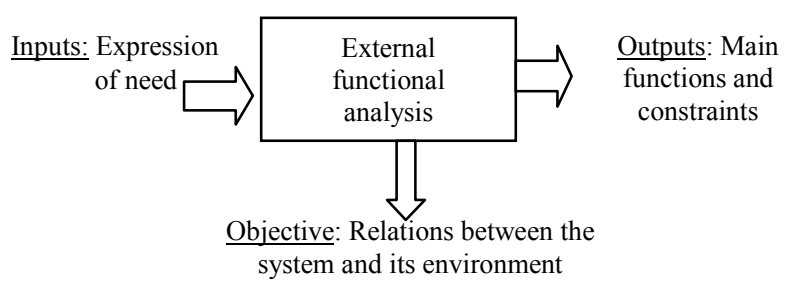

Fig. 2: External functional analysis

The external functional analysis illustrates the relationship between the system and its environment. The external functional analysis is built according to the following steps:

- Identification of the elements of the external environment

- Identification of the main functions and constraints (Octopus Diagram)

- Characterization of the main functions and constraints (thermal characteristics, electrical, mechanical, etc.).

- Definition of the mission profile of the system

After this analysis, the application of an internal functional analysis is necessary to determine the internal functions of the system. 


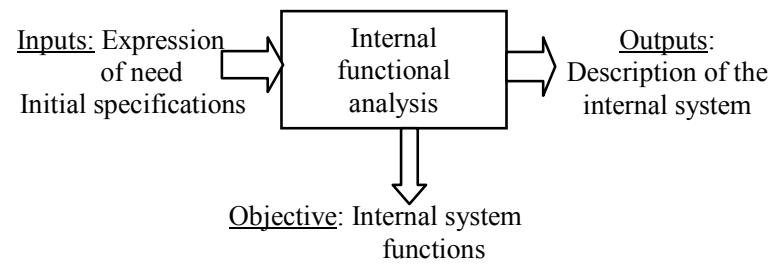

Fig. 3: Internal functional analysis

The internal functional analysis identifies the internal functions that are necessary to achieve the main functions of the system. There are several methods to achieve this analysis: we include for example, SADT, FAST, SysML, Functional tree, etc.

\section{Organic analysis}

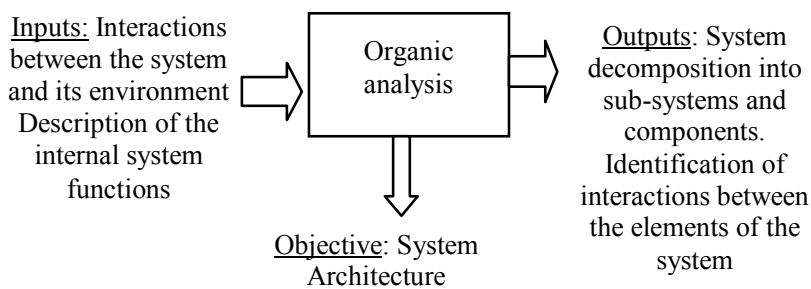

Fig. 4: Organic analysis

The objective of the organic analysis is to define the architecture of the system, the decomposition into sub-systems and components and the identification of the interactions between the different elements of the system.

We define the term "interaction" as the dependence between the elements of the system causing a change in the system reliability. The interaction can be functional or collateral.

Organic analysis is built according to the following steps:

- Definition of the components associated with internal functions (internal functional analysis)

- Building of the organic system architecture

- Decomposition of the system into sub-systems and components

- Identification of the functional interactions between the components of the system (functional interactions matrix)

- Identification of the collateral interactions between the components of the system (physical location of the components)

\section{Dysfunctional analysis}

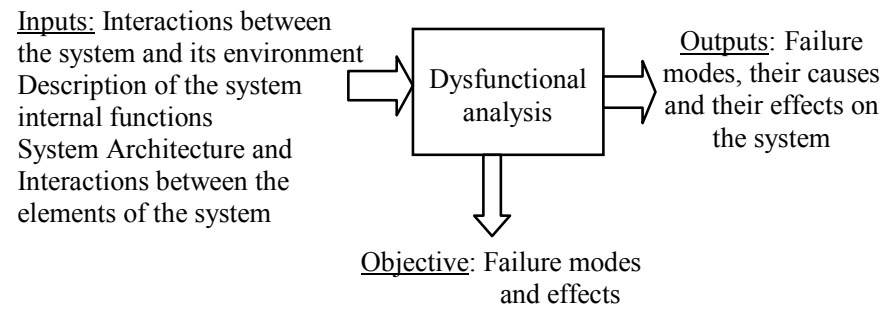

Fig. 5: Dysfunctional analysis

The objective of this step is to identify the dysfunctional failure modes and degradation of the system components and to analyze their effects on the system. To achieve the dysfunctional analysis, the outputs of functional and organic analyzes are needed. In other words, the outputs of functional and organic analyzes are the inputs of dysfunctional analysis.

The main methods used in a dysfunctional analysis are: the Preliminary Risk Analysis (PRA), Failure Modes, Effects and Criticality Analysis (FMECA), Analysis of the Effects of Software errors (AEEL), Trees Failures (ADD), etc [1]. The majority of these methods are adapted only to the hardware (mechanical, electronic, etc.); yet the AEEL is an equivalent of the FMECA that fits perfectly to the software part. The combination of these two techniques (FMECA + AEEL) can process the dysfunctional analysis of a mechatronic system.

For FMECA, we can add a column that specifies the type of damage (intrinsic, collateral or functional) and therefore information about its nature (first or second), in order to take into account the interactions between the components of the system.

\section{E. Qualitative modelling}

\begin{tabular}{|c|c|c|}
\hline $\begin{array}{l}\text { Inputs: Interactions between the } \\
\text { system and its environment } \\
\text { Description of the system } \\
\text { internal functions }\end{array}$ & $\begin{array}{l}\text { Qualitative } \\
\text { modelling }\end{array}$ & $\begin{array}{l}\text { Outputs: } \\
\text { Functional and } \\
\text { dysfunctional } \\
\text { models }\end{array}$ \\
\hline $\begin{array}{l}\text { System Architecture and } \\
\text { nteractions between the elements of } \\
\text { the system } \\
\text { Failure modes, their causes and } \\
\text { their effects on the system }\end{array}$ & $\begin{array}{l}\text { Objectiv } \\
\text { dysfunctio }\end{array}$ & $\begin{array}{l}\text { onal and } \\
\text { vior of the }\end{array}$ \\
\hline
\end{tabular}

Fig. 6: Qualitative modelling

The objective of the qualitative analysis is to model the functional and dysfunctional behaviours of the system and its components. To achieve this analysis, we rely on the various analyzes carried out previously into the different steps of the 
proposed approach and particularly functional, dysfunctional and organic analysis. The study of the FMECA method, enhanced by the classification of failure modes of components depending on their nature (first or second), their speed event (sudden, progressive) and their amplitude (partial or complete) allowed us to introduce new failure modes and additional states. Indeed, the failure modes commonly used in modelling are intrinsic failure modes of components (failure of first type as classified by nature). To take into account the interactions inducing collateral damage, the failure modes of second type must be added.

There are several methods that seem to be adapted for modelling mechatronic systems. We can mention: Petri nets [2], Markov chains [5], dynamic Bayesian networks (DBN) [6], neural networks [4], etc.

The most appropriate methods for modelling mechatronic systems are state-transition models such as state graphs (Markov graphs, Bayesian networks) and approaches based on Petri nets [3]. In this study, we chose to use Petri nets because they allow:

- $\quad$ The modelling of mechatronic systems integrating different technologies

- $\quad$ The use in each stage of the development cycle

- The analysis of functional and dysfunctional behaviour

- The modelling of continuous and discrete events (hybrid systems)

- Taking into account the dynamic behaviour of the system

- The modification of their internal structures ( reconfigurability)

- $\quad$ The specification of interactions between the components.

One of the drawbacks of Petri nets could limit its application in industry due to the lack of use.

To illustrate the use of Petri nets, let's imagine a system where a bearing and a coil are placed so that a collateral interaction exists between them, due to the bearing heating after 6 millions operating cycles [12].

Figure 7 presents the functional and dysfunctional model of the coil.

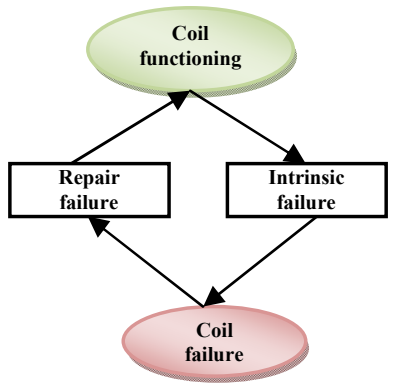

Fig. 7: Functional and dysfunctional model of the coil [12]
To model the coil, we considered three states (functioning, degraded and failure), failure mode (intrinsic failure) and a repair mode (repair failure). When the coil is considered alone, the degraded state does not appear because it is induced by the interaction bearing / coil. The interaction is not considered in this first model, the transition from one operating state to a fault state occurs only following the occurrence of the failure mode "intrinsic failure". The mode "repair failure" allows you to operate the coil again.

Figure 8 presents the functional and dysfunctional model of the guide bearing.

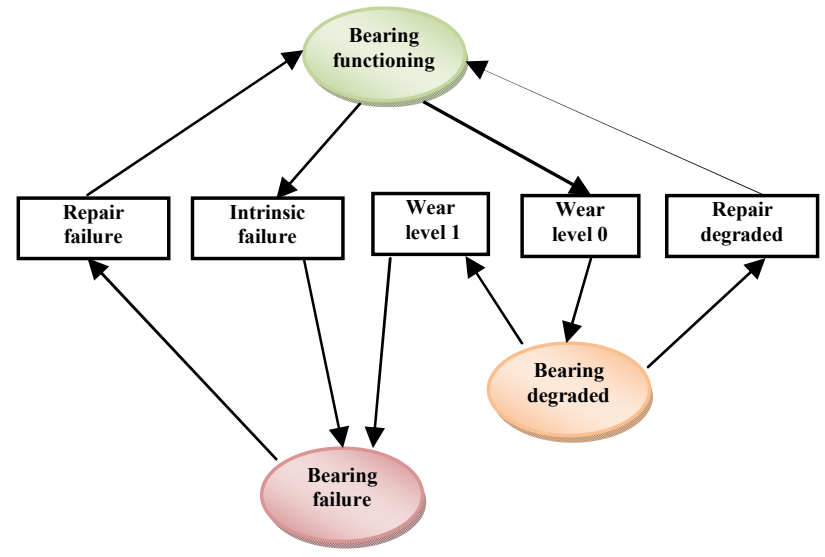

Fig. 8: Functional and dysfunctional model of the bearing [12]

To model the bearing, we considered three states (functioning, degraded and failure), three failure modes (intrinsic failure, wear level 0, and wear level 1) and two modes of repair (repair failure and repair degraded).

Figure 9 presents the functional and dysfunctional model of the guide bearing and the coil taking into account the interaction Bearing /Coil. The degraded state of the bearing has a direct impact on the functioning of the coil. Indeed, for a temperature higher than the nominal temperature (for example $120{ }^{\circ} \mathrm{C}$ ), reached due to overheating of the bearing in degraded mode, the operation of the coil becomes abnormal (coil degraded state). Over time the degraded state of the coil will induce a failure of the latter due to its overheating.

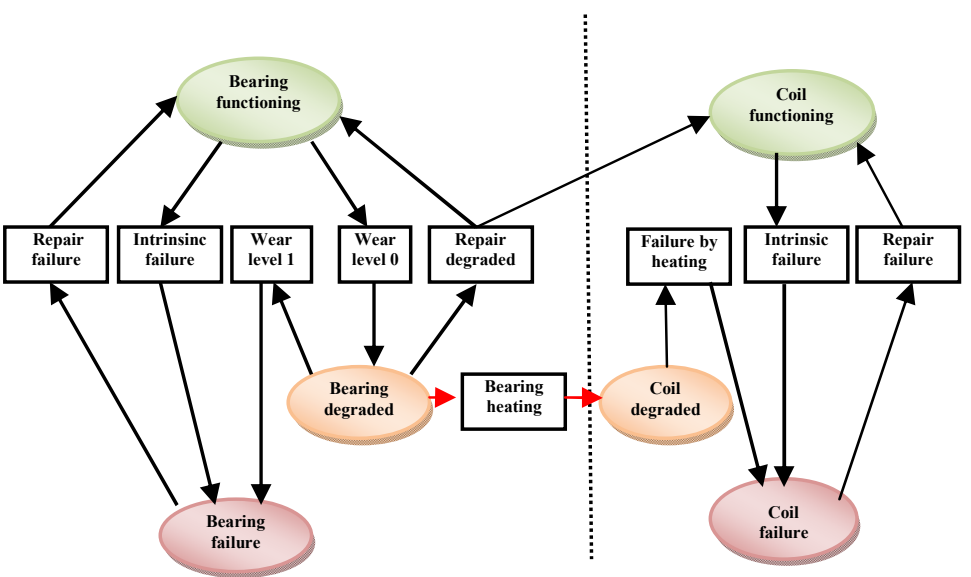

Fig. 9: Functional and dysfunctional model of the bearing and the coil [12] 
Taking into account the interaction between the two components, it was necessary to define new states and failure modes: a state of partial amplitude called "coil degraded" and two failure modes second called "bearing heating" and "failure by heating."

The state "bearing degraded" induces the heating of the bearing that will put the coil in the state "coil degraded."

The "repair degraded" mode of the bearing resets the operating mode of the coil and of the bearing.

The state "failure by heating" of the coil is a mode so that complete degradation thereof leads to a fault state.

\section{F. $\quad$ Gathering and processing of data for the components}

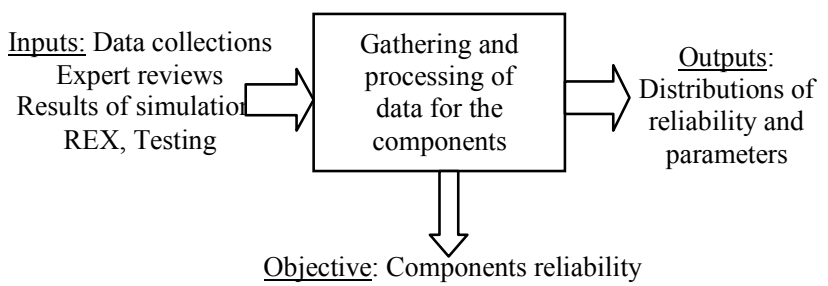

Fig. 10: Gathering and processing of the data for the components

The objective of this step of the methodology is to identify the laws of reliability associated with the components and then to gather and process the data in order to calculate their parameters.

Table 1 presents the distributions of reliability and their parameters for each type of technology.

\begin{tabular}{|c|c|c|}
\hline Technology & $\begin{array}{c}\text { Reliability } \\
\text { distributions }\end{array}$ & Parameters \\
\hline Electronic & Exponential & $\lambda$ \\
\hline Electrical & Exponential & $\lambda$ \\
\hline Mechanical & Weibull & $\beta$ and $\eta$ \\
\hline Software & Exponential & $\begin{array}{c}\lambda\left(\lambda=k p N_{0}\right. \\
\text { Musa model })\end{array}$ \\
\hline
\end{tabular}

Table 1: Reliability distributions associated with the technology of components

\section{$\lambda$ : Failure rate}

$\beta$ : Shape Parameter

$\eta$ : Scale Parameter

$\mathrm{k}$ : Constant that depends on the dynamic structure of the program

p: Number of executions per time unit

$\mathrm{N}_{0}$ : Initial number of faults in the program
The most databases used for electronic components are FIDES, MIL-HDBK 217F, and RDF 2000. We are interested in our approach to FIDES model that can take into account the mission profile of the product.

Calculating the failure rate of electronic components using FIDES database

The expression of the failure rate depends on several factors: design technology, manufacturing technology and environmental operation of the component. Then, according to FIDES, the failure rate depends on a basic failure rate of component, weighted by factors of technology, design, manufacture, use, environment, etc. [7]. The failure rate according to FIDES can be written as follows:

$$
\lambda=\lambda_{\text {Physical }} * \prod_{\text {Part_Manufacturing }} * \Pi_{\text {Process }} * \Pi_{\mathrm{RF}-\mathrm{HF}} * \Pi_{\mathrm{H}-\mathrm{M}} * \Pi_{\mathrm{LF}}
$$

\section{Where}

$\lambda$ : Predicted failure rate;

$\lambda_{\text {Physical }}$ : Represents the physical contribution;

$\Pi_{\text {Part_Manufacturing: Reflects the quality and technical control }}$ of component manufacturing;

$\Pi_{\text {process: }}$ : Reflects the quality and technical control of the processes of developing, manufacturing and use of products containing the component;

$\Pi_{\mathrm{RF}-\mathrm{HF}}$ : Reflects the quality and control of the life cycle of the product radiofrequency (RF) or high frequency (HF);

$\Pi_{\mathrm{H}-\mathrm{M}}$ : Reflects the quality and control of design and manufacture of hybrid or MCM (Multi Chip Modules);

$\Pi_{\mathrm{LF}}$ : Reflects the factor of transition to lead-free processes;

The method for calculating the failure rate of electronic components according to FIDES includes three steps:

- Defining the mission profile (phases, duration, temperature, humidity, vibration, etc.)

- $\quad$ Calculating the values of $\Pi$ factors $\left(\prod_{\text {process, }} \prod_{\mathrm{PM}}\right.$, $\prod_{\text {placement, }}, \mathrm{C}_{\text {sensitivity }}, \prod_{\text {application, }} \prod_{\text {ruggedising, }} \prod_{\mathrm{RF}-\mathrm{HF}}, \prod_{\mathrm{H}-\mathrm{M} \text {, }}$ $\left.\Pi_{\mathrm{LF}}\right)$

- Calculating the failure rates (EASYREL tool for example)

\section{G. Modelling and simulation}

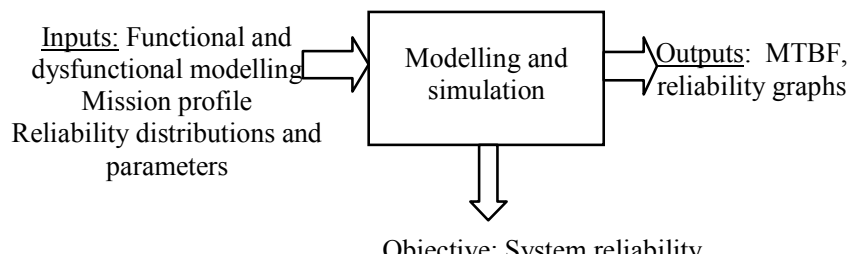

Fig. 11: Modelling and simulation 
The objective of this step is to estimate the reliability for the system and its components over the time of use and according to the mission profile of the system. Two methods are used:

- Petri Nets for behavioural modelling (functional and dysfunctional) and Monte Carlo simulation for convergence of results.

- Modified Reliability Block Diagrams, adapted in order to take into account the mission profile and the considered interactions for exact calculation.

The comparison of the results obtained by the two methods is necessary to verify and validate on the one hand, the model of Petri nets and the parameters chosen for simulation, and on the other hand, the calculation method developed in the context of reliability block diagrams considering the mission profile and interactions.

The modelling and simulation are organized according to the following three steps:

- Implementation of the quantitative models according to the qualitative analysis,

- Considering of reliability laws for components and their parameters (models without interaction),

- Considering and modelling of the interactions and their hypothesis (models with interaction).

\section{CONCLUSION}

In this article, we analyzed some of the problems associated with the reliability of mechatronic systems and we proposed an overall methodology evaluating the predictive reliability that takes into account the specificities of these systems.

To evaluate the predictive reliability of a mechatronic system, it is necessary to have a model because the system does not exist at this stage of the development and cannot be physically tested. That is why the first part of the proposed methodology is a qualitative analysis that provides all the necessary information on the functioning and malfunctioning of a mechatronic system. The second part of the methodology is a quantitative analysis to estimate and quantify the reliability of the mechatronic system.
We validated this methodology through industrial mechatronic examples. These examples are selected in collaboration with our partner CETIM which has very close relations with many companies working in the field of mechatronics. Also, we deepened our analysis on more theoretical questions, to respond in particular to the problems of specification and modelling, collateral and functional interactions in terms of reliability and to evaluate the influence of the mission profile of the distribution laws for component reliability.

\section{Acknowledgment}

We thank the ACS (Assembly of the Countries of Savoie) and CETIM that fund this work as part of a doctoral thesis.

\section{References}

[1] A. Mihalache; Modeling and evaluation of the reliability of mechatronic systems: application on embedded system; PhD thesis, University of Angers, 2007.

[2] G. Moncelet; Application of Petri nets to evaluate the dependability of mechatronic systems in the automotive world; $\mathrm{PhD}$ thesis, University Paul Sabatier Toulouse, October 1998.

[3] M. Medjoudj; Contribution to the analysis of systems controlled by computers: Extraction scenarios feared and verification of time constraints; PhD thesis, University Paul Sabatier Toulouse, 2006.

[4] S. Piechowiak; Artificial intelligence and diagnosis, Technical Engineering, page 20, December 2003.

[5] A. Villemeur; Safe operation of industrial systems. Editions, 1988.

[6] S. Verron. Diagnosis and monitoring of complex processes by Bayesian networks. PhD thesis, ISTIA - University of Angers, 2007.

[7] France Airbus, Eurocopter, Nexter Electronics, MBDA France, Thales Airborne Systems, Thales Avionics, Thales Research \& Technology and Thales Underwater Systems, FIDES Guide 2009, under the supervision of the General Delegation for Armaments May 2009.464 pages.

[8] R.W Daniel,, J.R. Hewit, Editorial. Mechatronics, 1(1): i-ii, 1991

[9] .C. Commault, J.M. Dion, A. Perez, Disturbance rejection for structured systems. IEEE Transactions on Automatic Control, 36(7):884-887, juillet 1991.

[10] AFNOR. Mechatronics - vocabulary. NF E01-010, November 2008.

[11] A. Demri; Contribution to the assessment of the reliability of a mechatronic system by functional and dysfunctional modeling; $\mathrm{PhD}$ thesis, University of Angers, 2009.

[12] N. Chouket G. Habchi C. Barthod. Implementation of a methodology for evaluation of the reliability of mechatronic system. Application for smart actuator, internal report, Univ. Savoie, March 2014 